\title{
Male Infertility Effective Factors and Failure Type Determination in Iranian Infertile Men
}

\section{ART ICLE INF O}

\section{Article Type}

Original research

\section{Authors}

Saremi A.T.* $M D$

Zamanian M. ${ }^{1} M D$

Pooladi A. ${ }^{2} M D, P h D$

\section{How to cite this article}

Saremi A T, Zamanian M, Pooladi

A. Male Infertility Effective Facto-

rs and Failure Type Determination

in Iranian Infertile Men. Sarem

Journal of Reproductive Medicine. 2018;2(1):3-8
*"Sarem Fertility \& Infertility Research Center (SAFIR)" and "Sarem Cell Research Center (SCRC)", Sarem Women's Hospital, Tehran, Iran ${ }^{1}$ Sarem Women's Hospital, Tehran, Iran

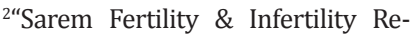
search Center (SAFIR)" and "Sarem Cell Research Center (SCRC)", Sarem Women's Hospital, Tehran, Iran

\section{Correspondence}

Address: Sarem Women's Hospital, Basij Square, Phase 3, Ekbatan Town, Tehran, Iran. Postal Code: 1396956111

Phone: +98 (21) 44670888

Fax: +98 (21) 44670432

saremiat@yahoo.com

\section{Article History}

Received: September 27, 2016

Accepted:December 20,2016

ePublished: February 15, 2018

\section{A B S T RA C T}

Aims The evaluation of effective factors on male infertility is essential to diagnose and to treat the causes of infertility. The aim of this study was to investigate the factors affecting infertility and to determine the type of sperm disorders in Iranian infertile men.

Materials \& Methods This cross-sectional community-based study was conducted on 764 Iranian infertile men referred to the infertility clinic of Sarem women's hospital (Tehran, Iran) from April 2006 to March 2012. The patients' demographics, history of disease, surgery, and seminal fluid analysis were obtained from their medical records. The semen samples were collected according to the WHO standard protocol. The data were analyzed by Chi square test using SPSS 16 software.

Findings The pure male factor infertility was found in $470(61.5 \%)$ patients and $294(38.5 \%)$ patients showed male factor infertility associated with other factors (mixed male factor infertility). Primary infertility was found in 593 (77.6\%) patients and 171 (22.4\%) patients showed secondary infertility. Asthenospermia and asthenoteratospermia were found in $236(30.9 \%)$ and 225 (29.5\%) patients, respectively. The prevalence of asthenospermia and asthenoteratospermia were higher than the other sperm disorders in the patients. The government employees and self-employed person were more frequent than the patients with other jobs. There was no significant relationship between the job status and male infertility $(p>0.05)$. The most of the patients were from Tehran and central provinces of Iran.

Conclusion Demographics, occupational factors, varicocele disease history, and type of surgeries are not related to infertility. The prevalence of asthenospermia and asthenoteratospermia among Iranian infertile men are more than the other sperm disorders. bone marrow is fractal.

Keywords Epidemiology; Infertility; Male Infertility

\section{CIT A T I O N L INKS}

[1] WHO manual for the standardized investigation and diagnosis of the infertile ... [2] The decline of infertility: Apparent ... [3] Evidence-based management of male ... [4] Proximal tubal disease: The place for tubal ... [5] Effect of female age on the diagnostic categories of ... [6] Epidemiology and management of ... [7] Incidence and main causes of infertility in a resident population ... [8] Semen quality and age-specific changes: A study between two ... [9] Semen quality in fertile US men in relation to geographical ... [10] Sexual, marital, and social impact of a man's perceived ... [11] Evaluation of the subfertile ... [12] Clinical relevance of oxidative stress in male ... [13] Chronic prostatitis and male accessory gland infection--is there an impact on ... [14] Semen analysis in laboratory practice: An overview ... [15] Decreased protein tyrosine phosphorylation and membrane ... [16] Is varicocelectomy indicated in subfertile men ... [17] Chronic orchitis: A neglected cause of male ... [18] Reproductive hazards of fire ... [19] Sexually transmitted infections: Impact ... [20] Testicular cytology in ... [21] Endocrine correlates of infertility in male ... [22] New method of evaluating sperm morphology with ... [23] Evidence for decreasing quality of semen ... [24] Evidence of deteriorating semen quality in the United ... [25] Caffeinated and alcoholic beverage intake in relation to ... [26] Occupationally related exposures ... [27] Semen quality in a population of volunteers ... [28] Prognostic value of meiotic spindle imaging ... [29] Effect of smoking on seminal plasma ascorbic ... [30] Is varicocelectomy indicated in subfertile men ... [31] Oxidative stress and medical antioxidant... [32] Improving reproductive performance in overweight/obese ... [33] Clinical laboratory evaluation of male ... [34] The analysis of the sperm parameters in view of its concentration and ... [35] Factors associated with male ... [36] Male factor infertility: Five years ... [37] Prevalence of low sperm count and abnormal ... [38] Sonographic spectrum of scrotal ... [39] Contribution of environmental ... [40] factors ... Leukocytospermia in male infertility patients ... [41] Lifestyle and environmental ... 


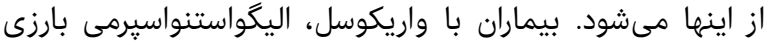

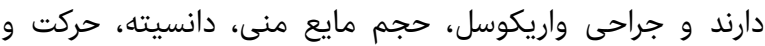

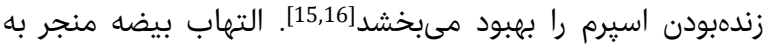

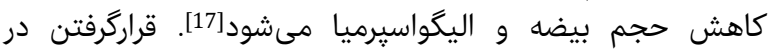

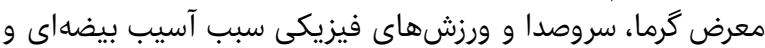

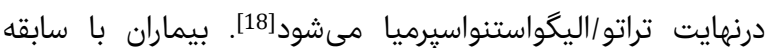

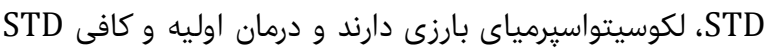

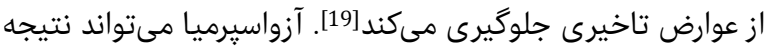

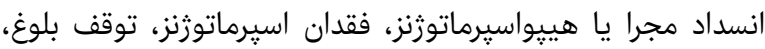

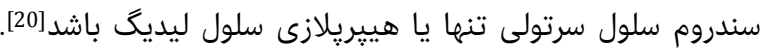

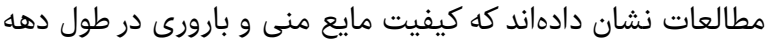

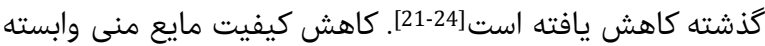

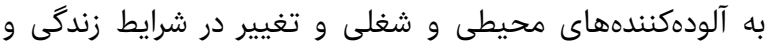

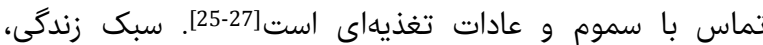

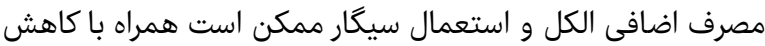

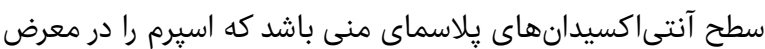

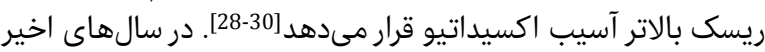

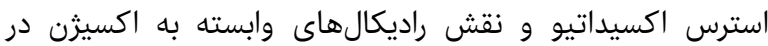

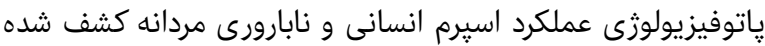

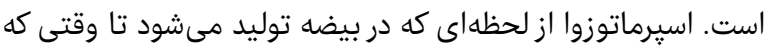

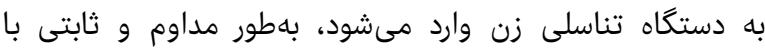

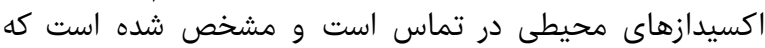

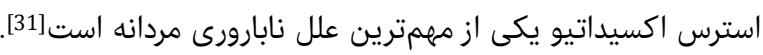

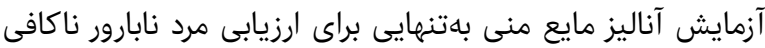

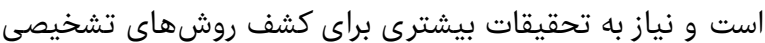

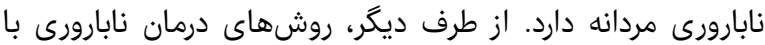

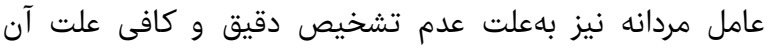

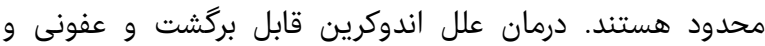

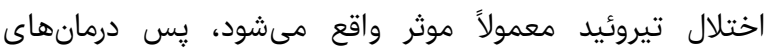

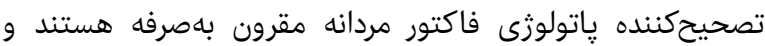

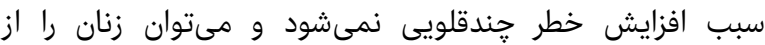

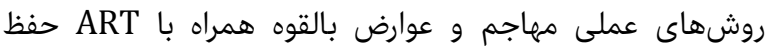

از آنجايى كه شيوع فاكتورهاى غيرقابل توجيه نابارورى مانند

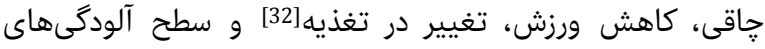

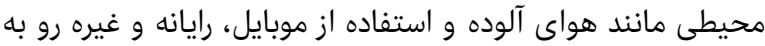

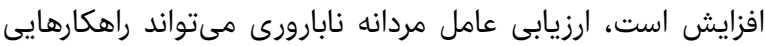

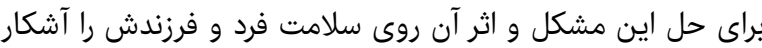

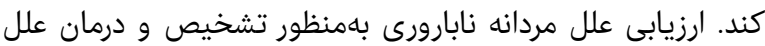

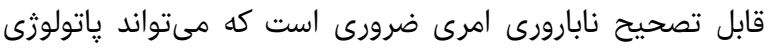

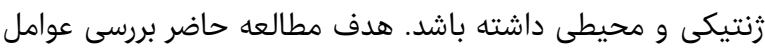

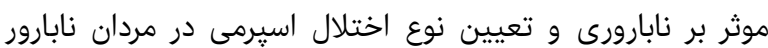

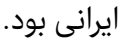

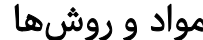

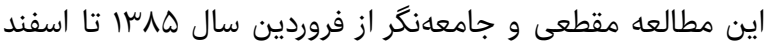
.

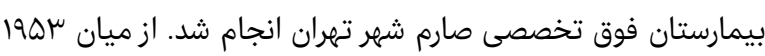

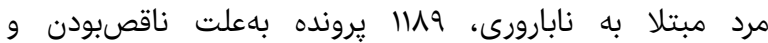

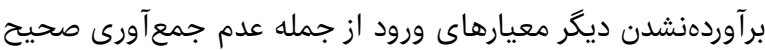

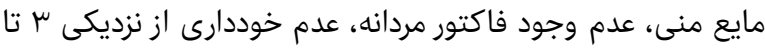

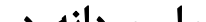

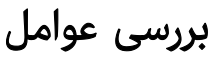

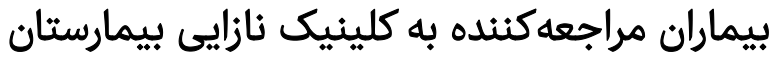

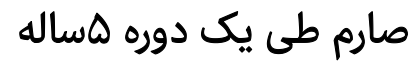

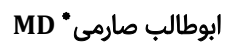

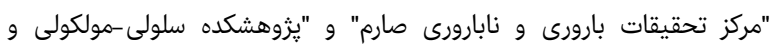

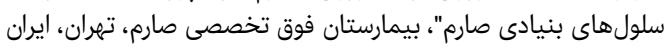

MD مرضيه زمانيان

بيمارستان فوق تخصصى صارم، تهران، ايران

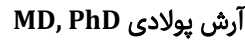

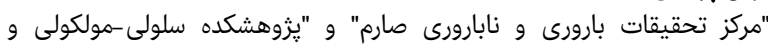

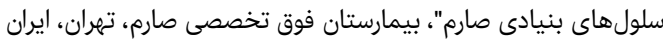

جكيده

اهداف: ارزيابى عوامل موثر بر نابارورى مردان بهمنظور تشخيص و درمان علل

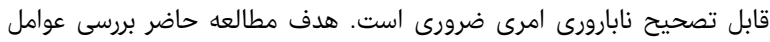

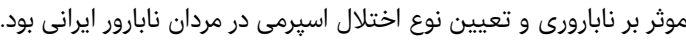

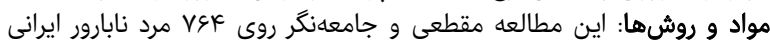

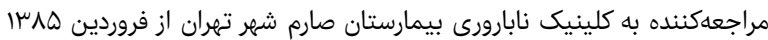

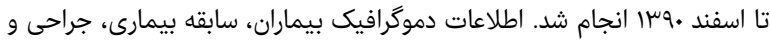

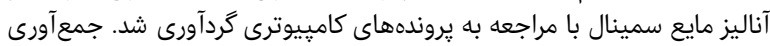

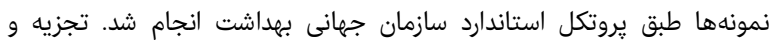

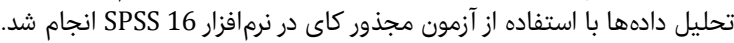

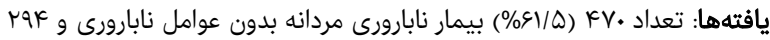
(ب⿳N/A)

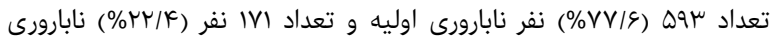

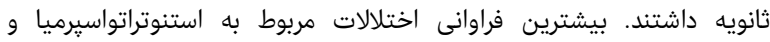

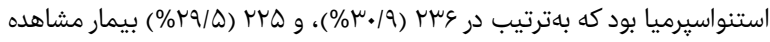

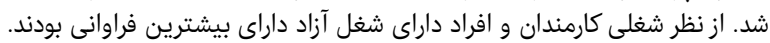

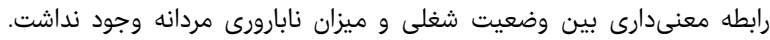

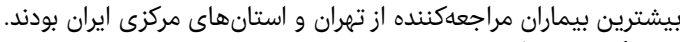

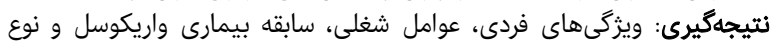

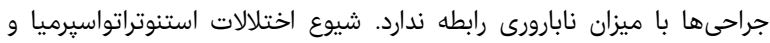

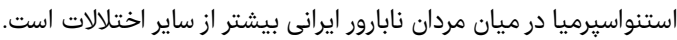
كليدوازهها: عوامل محيطى، نابارورى، عامل مردانه نابارورى إنياني

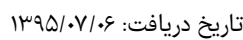

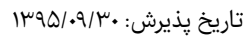

"نويسنده مسئول: saremiat@yahoo.com

مقدمه

نازايى بهعنوان عدم توانايى در باردارشدن بعد از يكسال نزديكى

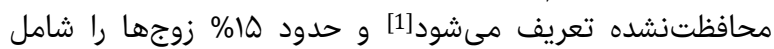

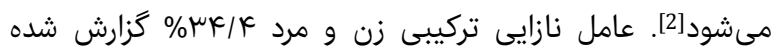

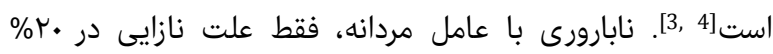

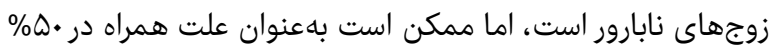

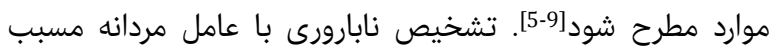

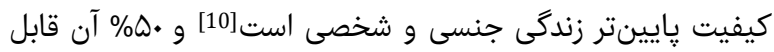

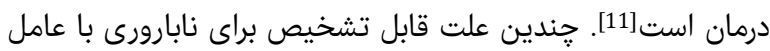

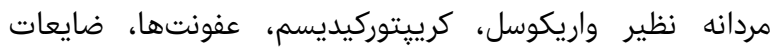

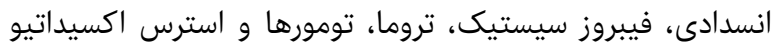

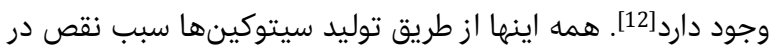

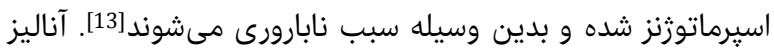

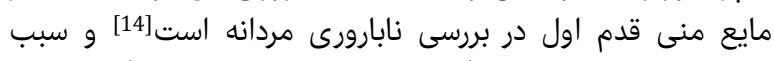

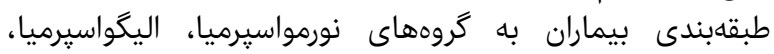

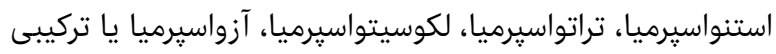




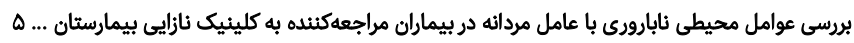

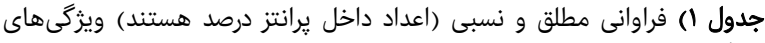

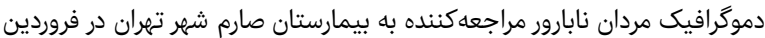

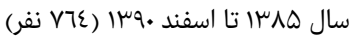

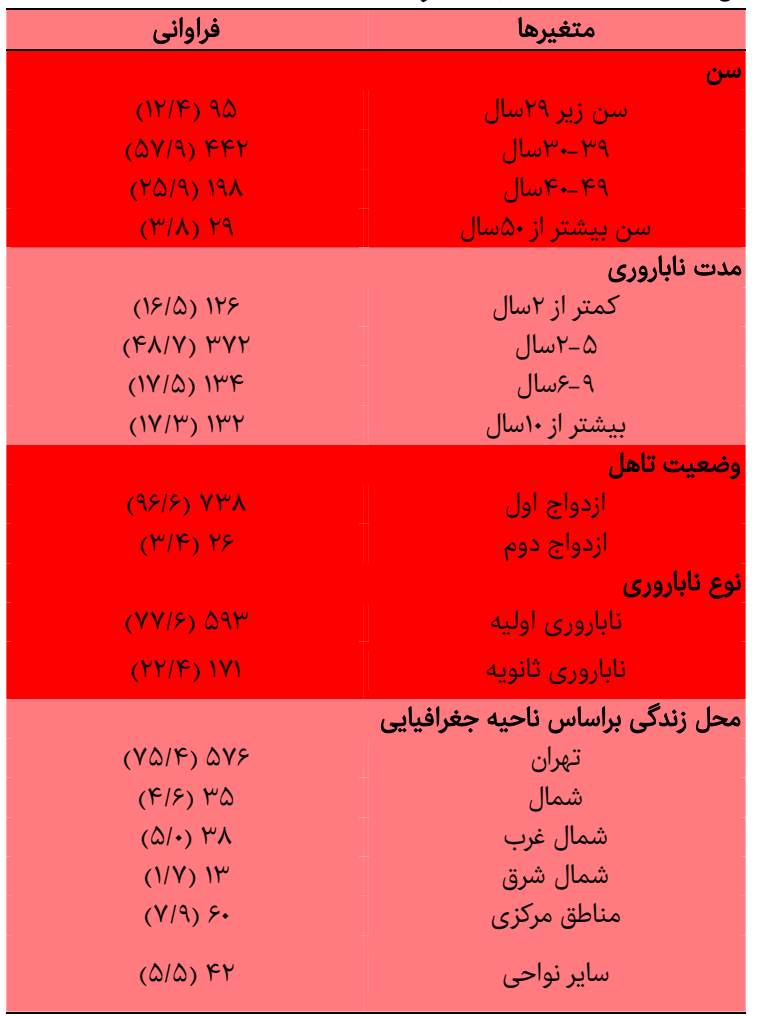

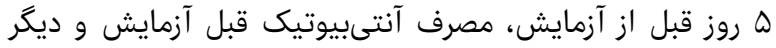

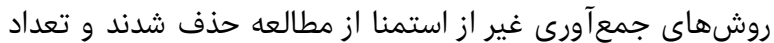

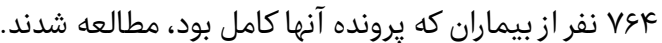

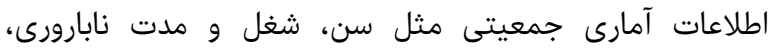

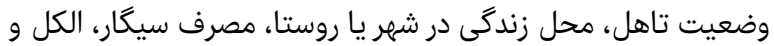

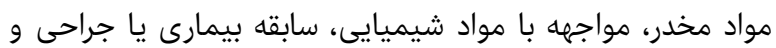

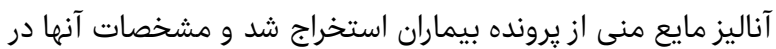

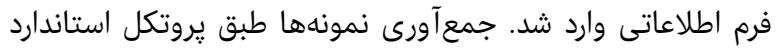

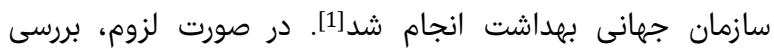
هورمونى و ارجاع به اندرولوزيست نيز انجام شاندام شدان

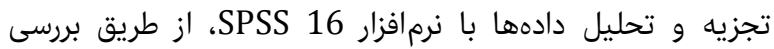
ميانكين دادهها و آزمون مجذور كاى انجام شد. نار

\section{يافتهها}

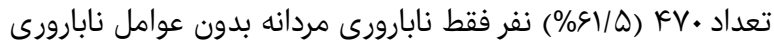
(ن) نفر مبتلا به (pure male factor)

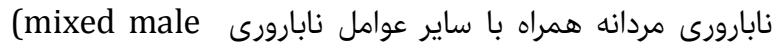
بودند. ميانكين سنى بيماران factor)

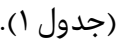

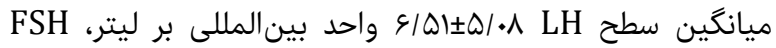

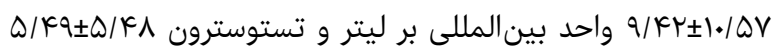

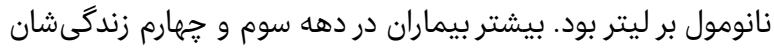

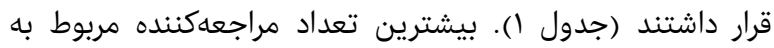
محدوده سنى q

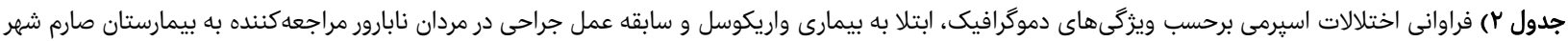

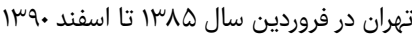

\begin{tabular}{|c|c|c|c|c|c|c|}
\hline اليكواستنوتراتواسيرميا & اليكواستنواسيرميا & استنوتراتواسيرميا & اليكواسيرميا & آزواسيرميا & استنواسيرميا & متغيرها \\
\hline$(r \cdot \mid Q) \mid Q V$ & $(F / \Lambda)^{\mu V}$ & $(\mu / q) r \mu q$ & $(T / M) 18$ & $(I r / T) q \mu$ & $(r / / \Delta) r r \Delta$ & فراوانى كل \\
\hline & & & & & & سن (سال) \\
\hline$r \Lambda$ & k & 11 & - & iv & rA & زير M \\
\hline 19 & 10 & 10 . & 9 & $\Delta r$ & ITV & $\mu \cdot-\mu q$ \\
\hline$\mu \Delta$ & IV & $\Delta \Lambda$ & c & r. & qr & $k \cdot-k q$ \\
\hline$\Delta$ & 1 & 1. & 1 & k & $\wedge$ & بيشتر از•ه \\
\hline gr & ז & 1.9 & 9 & ץ & $q$ & كارمند دولتى \\
\hline द9 & M & ᄉ1 & v & $\mu \Delta$ & १९ & آزاد \\
\hline k & . & $\pi$ & . & r & v & راننده \\
\hline$\wedge$ & r & 1. & • & \& & ir & كارگر ساده \\
\hline$\wedge$ & . & द & • & $\Delta$ & $\Delta$ & كارگر صنعتى \\
\hline$\mu$ & • & r & • & $r$ & • & كشاورز \\
\hline . & • & 1 & . & . & r & نانوا \\
\hline r & . & 9 & . & . & r & نظامى \\
\hline$\mu$ & . & v & . & k & \& & مهندس \\
\hline 1 & 1 & r & . & 1 & r & يزشك \\
\hline & & & & & & واريكوسل \\
\hline 11 & r & 1. & r & 9 & $\mathbb{1}$ & درجه | \\
\hline$\mu v$ & 8 & $\mu F$ & . & $\mu$ & $\mu$. & درجه r \\
\hline rV & 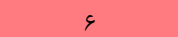 & kq & \& & $v$ & $\mu_{\Delta}$ & درجه س \\
\hline & & & & & & لـ زندكى \\
\hline $\begin{array}{c}10 \mu \\
k\end{array}$ & ${ }^{\mu} \gamma$ & ris & 19. & $\begin{array}{l}19 \\
k\end{array}$ & $\underset{r r \mu}{r \mu h}$ & شهر: \\
\hline & & & & & & نوع جراحى \\
\hline$k$ & $\cdot$ & 1 & $\cdot$ & $\mu$ & 9 & هيدروسلكتومى \\
\hline k & . & 1 & 1 & v & $\mu$ & اركيويكسى \\
\hline v & 1 & v & r & $k$ & v & فتق اينكوينال \\
\hline r & 1 & r & - & 9 & r & وازكتوكى - وازوستومى - اركيدكتوى \\
\hline$r$ & • & k & . & 1 & k & ساير \\
\hline
\end{tabular}


نابارورى، هيدروسل و فتق اينكوينال بيشترين ارتباط را با

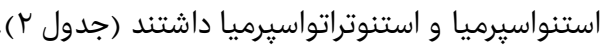

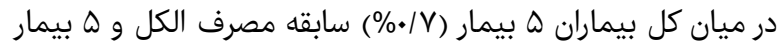

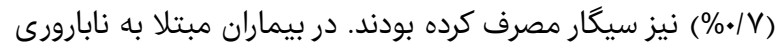

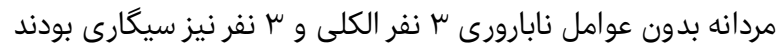

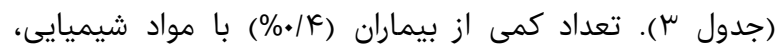
تركيبات هورمونى، سمى و مواد مخدر مواجها داشئ داشتند.

بحث

اثرات مضر احتمالى عوامل محيطى مثل گرما و عوامل شيميايى،

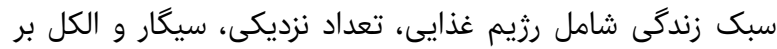

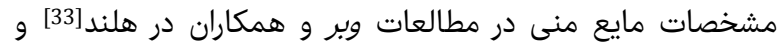

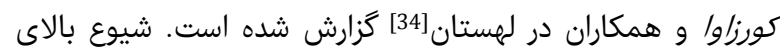

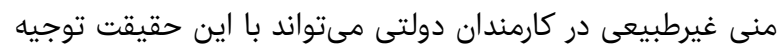

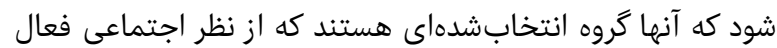

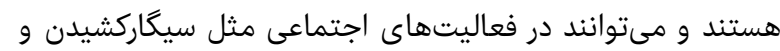

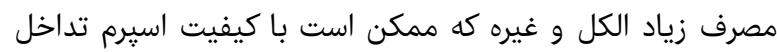

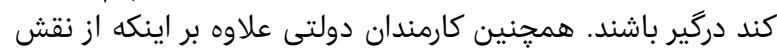

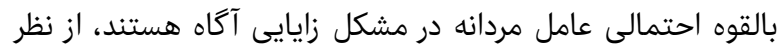

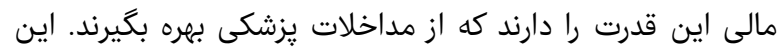

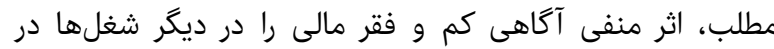

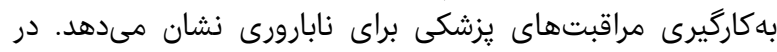

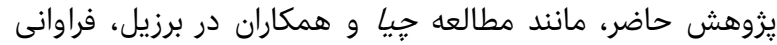

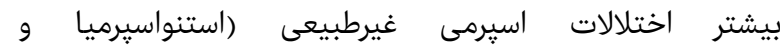

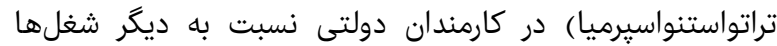

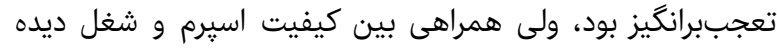

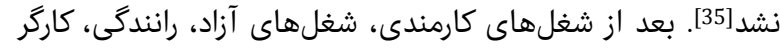

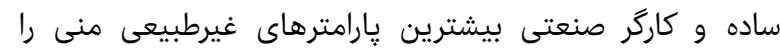

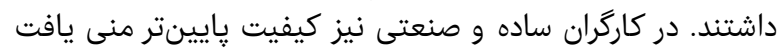

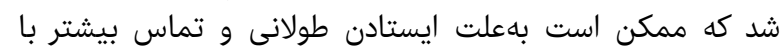

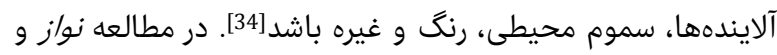

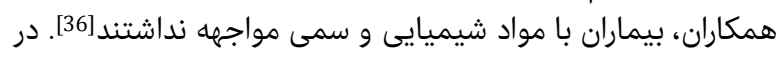

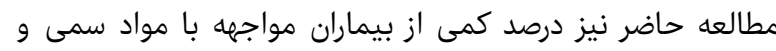

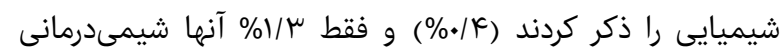

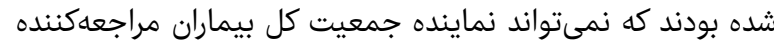

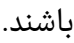

اين مطالعه از نظر شيوع بيشتر شغل كارمندى مشابه مطالعه

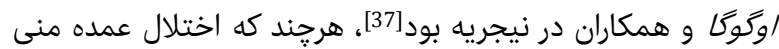

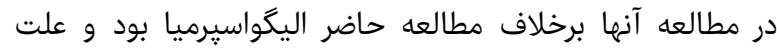

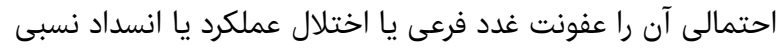

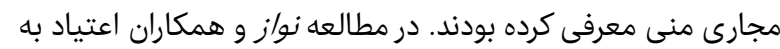

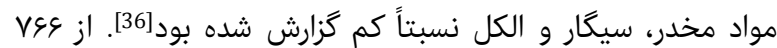

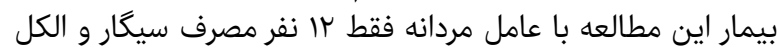

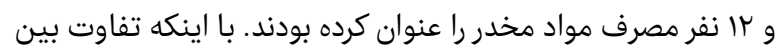

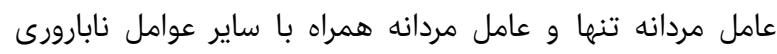

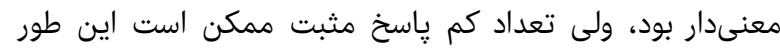

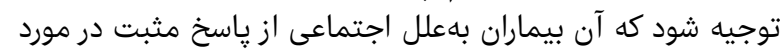

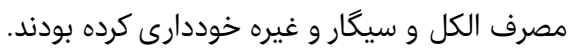

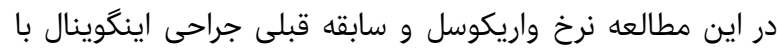

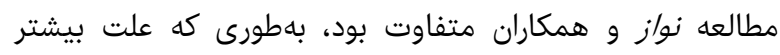

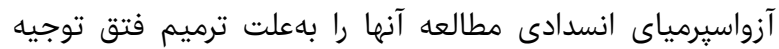
مىكرد[36]. مطالعه حاضر از نظر شيوع بالاتر واريكوسل مشابه ترميه

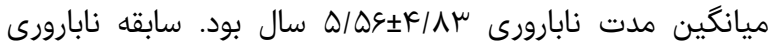

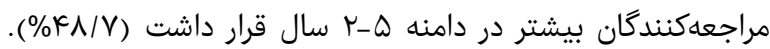

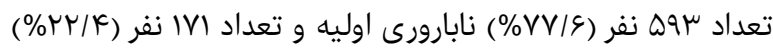

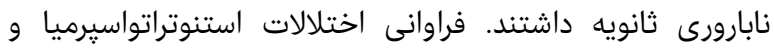

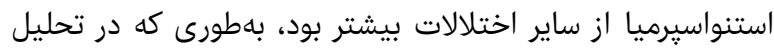

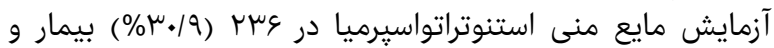

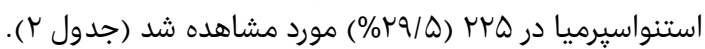

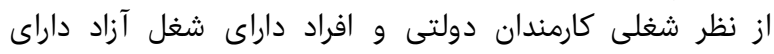

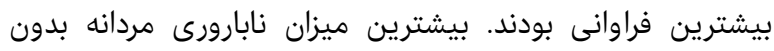

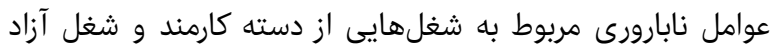

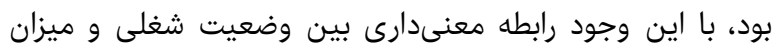

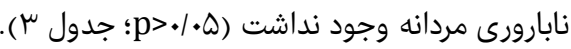

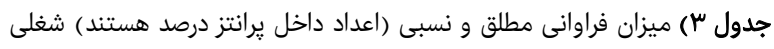

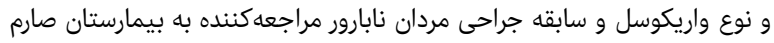

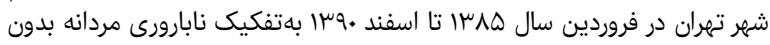

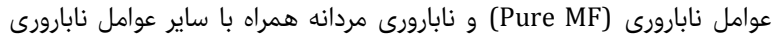

\begin{tabular}{|c|c|c|c|}
\hline Mixed MF & Pure MF & فراوانى & متغيرها \\
\hline & & & واريكوسل \\
\hline $\mathbb{1 r}$ & MQ & $(1 / \varepsilon) \mathrm{kV}$ & لدرجه | \\
\hline mq & VI & $(\| F / \mu) \|$. & درجه r \\
\hline kq & 1) & $(\mid \varepsilon / r) I r V$ & درجه س \\
\hline $\mathbb{I r V}$ & $19 \mathrm{~V}$ & $\begin{array}{l}\text { HYK } \\
(K Y / F)\end{array}$ & شغل \\
\hline$\| \varepsilon$ & 19. & $(\kappa \cdot / 1)^{\mu \cdot \varphi}$ & آزاد \\
\hline I & ir & $(\mu / \mu) r \Delta$ & 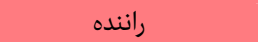 \\
\hline ir & re & $(\Delta / \cdot) \Psi_{\Lambda}$ & كارگر ساده \\
\hline$\Delta$ & 19 & $(\mu / I) \Gamma F$ & كارگر صنعتى \\
\hline$r$ & $\Delta$ & $(१ / \bullet) \vee$ & كشاورز \\
\hline . & $\mu$ & $(\cdot / k) \mu$ & نانوا \\
\hline \& & $k$ & $(1 / \mu) 1$. & 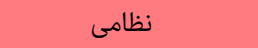 \\
\hline 9 & 11 & $(r / \varepsilon) r$. & مهندس \\
\hline k & $\mu$ & $(\cdot / 9) V$ & يزشك \\
\hline$\Delta$ & $q$ & $(1 / \Lambda) \mathbb{k}$ & نوع جراحى هـروسكتومى \\
\hline$\mu$ & שו & $(Y / I) \mid \varepsilon$ & اركيويكسى \\
\hline ז & 10 & $(r / V) r \Lambda$ & فتق اينگوينال \\
\hline k & ir & $(r / 1) \notin \varepsilon$ & وازكتومى - وازوستومى - \\
\hline - & 10 & $(Y / \cdot) 10$ & سابقه بيويسى از بيضه \\
\hline द & $\Delta$ & $(1 / F) \|$ & ساير \\
\hline
\end{tabular}

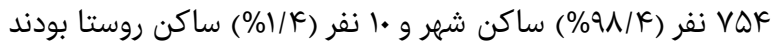

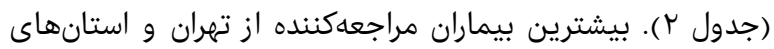

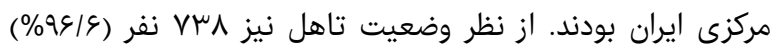

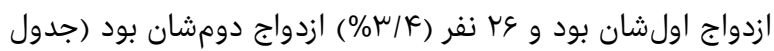

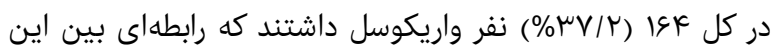

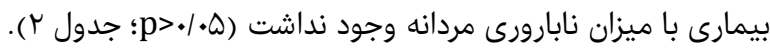

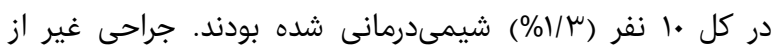

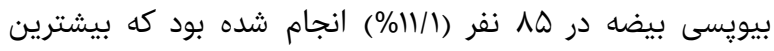

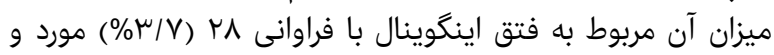

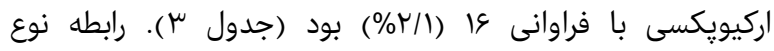

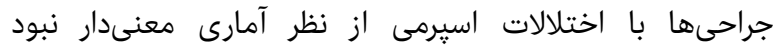

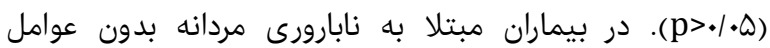


exposures and reduced semen quality: A case-control study. Fertil Steril. 1999;71(4):690-6.

27- Lopez Teijon M, Garcia F, Serra O, Moragas M, Rabanal A, Olivares $R$, et al. Semen quality in a population of volunteers from the province of Barcelona. Reprod Biomed Online. 2007;15(4):434-44.

28- Braga DP, Figueira Rde C, Rodrigues D, Madaschi C, Pasqualotto FF, Iaconelli A Jr, et al. Prognostic value of meiotic spindle imaging on fertilization rate and embryo development in in vitro-matured human oocytes. Fertil Steril. 2008;90(2):429-33.

29- Mostafa T, Tawadrous G, Roaia MM, Amer MK, Kader RA, Aziz A. Effect of smoking on seminal plasma ascorbic acid in infertile and fertile males. Andrologia. 2006;38(6):221-4.

30- Zhu Q, Meisinger J, Emanuele NV, Emanuele MA, LaPaglia N, Van Thiel DH. Ethanol exposure enhances apoptosis within the testes. Alcohol Clin Exp Res. 2000;24(10):1550-6.

31- Lanzafame FM, La Vignera S, Vicari E, Calogero AE. Oxidative stress and medical antioxidant treatment in male infertility. Reprod Biomed Online. 2009;19(5):63859.

32- Norman RJ, Noakes M, Wu R, Davies MJ, Moran L, Wang JX. Improving reproductive performance in overweight/obese women with effective weight management. Hum Reprod Update. 2004;10(3):267-80.

33- Weber RF, Dohle GR, Romijn JC. Clinical laboratory evaluation of male subfertility. Adv Clin Chem. 2005;40:317-64.

34- Kurzawa R, Kozanecka A, Glabowski W, Malinowska $\mathrm{D}$, Rozewicki S. The analysis of the sperm parameters in view of its concentration and motility in relation to men's age and occupation. Ginekol Pol. 1998;69(6):460. 35- Chia SE, Lim ST, Tay SK. Factors associated with male infertility: Sperm characteristics, strict criteria sperm morphology analysis and hypoosmotic swelling test. Int J Obstet Gynaecol. 2000;107(1):55-61.

36- Nawaz A, Rafiq M. Male factor infertility: Five years experience. Ann Pak Inst Med Sci. 2010;6(1):7-10.

37- Ugwuja EI, Ugwu NC, Ejikeme BN. Prevalence of low sperm count and abnormal semen parameters in male partners of women consulting at infertility clinic in Abakaliki, Nigeria. Afr J Reprod Health. 2008;12(1):6773.

38- Qublan HS, Al Okoor K, Al Ghoweri AS, Abu Qamar A. Sonographic spectrum of scrotal abnormalities in infertile men. J Clin Ultrasound. 2007;35(8):437-41.

39- Oliva A, Spira A, Multigner L. Contribution of environmental factors to the risk of male infertility. Hum Reprod. 2001;16(8):1768-76.

40- Wang AW, Politch J, Anderson D. Leukocytospermia in male infertility patients in China. Andrologia. 1994;26(3):167-72.

41- Sharpe RM. Lifestyle and environmental contribution to male infertility. Br Med Bull. 2000;56(3):630-42. 10- Smith J, Walsh T, Shindel A, Turek P, Wing H, Pasch L, et al. Sexual, marital, and social impact of a man's perceived infertility diagnosis. J Sex Med. 2009;6(9):2505-15.

11- Kolettis PN. Evaluation of the subfertile man. Am Fam Physician. 2003;67(10):2165-72.

12- Agarwal A, Makker K, Sharma R. Clinical relevance of oxidative stress in male factor infertility: An update. Am J Reprod Immunol. 2008;59(1):2-11.

13- Everaert K, Mahmoud A, Depuydt C, Maeyaert M, Comhaire F. Chronic prostatitis and male accessory gland infection--is there an impact on male infertility (Diagnosis and therapy)?. Andrologia. 2003;35(5):32530.

14- Andrade Rocha FT. Semen analysis in laboratory practice: An overview of routine tests. J Clin Lab Anal. 2003;17(6):247-58.

15- Buffone MG, Brugo Olmedo S, Calamera JC, Verstraeten SV, Urrutia F, Grippo L, et al. Decreased protein tyrosine phosphorylation and membrane fluidity in spermatozoa from infertile men with varicocele. Mol Reprod Dev. 2006;73(12):1591-9.

16- Okeke L, Ikuerowo O, Chiekwe I, Etukakpan B, Shittu 0 , Olapade-Olaopa 0 . Is varicocelectomy indicated in subfertile men with clinical varicoceles who have asthenospermia or teratospermia and normal sperm density?. Int J Urol. 2007;14(8):729-32.

17- Schuppe HC, Meinhardt A, Allam JP, Bergmann M, Weidner W, Haidl G. Chronic orchitis: A neglected cause of male infertility?. Andrologia. 2008;40(2):84-91.

18- Agnew J, McDiarmid MA, Lees PS, Duffy R. Reproductive hazards of fire fighting. I. Non-chemical hazards. Am J Ind Med. 1991;19(4):433-45.

19- Ochsendorf FR. Sexually transmitted infections: Impact on male fertility. Andrologia. 2008;40(2):72-5.

20- Verma AK, Basu D, Jayaram G. Testicular cytology in azoospermia. Diagn Cytopathol. 1993;9(1):37-42.

21- Resko JA. Endocrine correlates of infertility in male primates. Am J Primatol. 1982;3(Suppl 1):37-44.

22- Kruger TF, Acosta AA, Simmons KF, Swanson RJ, Matta JF, Veeck LL, et al. New method of evaluating sperm morphology with predictive value for human in vitro fertilization. Urology. 1987;30(3):248-51.

23- Carlsen E, Giwercman A, Keiding N, Skakkebaek NE. Evidence for decreasing quality of semen during past 50 years. BMJ. 1992;305(6854):609-13.

24- Irvine S, Cawood E, Richardson D, MacDonald E, Aitken J. Evidence of deteriorating semen quality in the United Kingdom: Birth cohort study in 577 men in Scotland over 11 years. BMJ. 1996;312(7029):467-71.

25- Chavarro JE, Rich-Edwards JW, Rosner BA, Willett WC. Caffeinated and alcoholic beverage intake in relation to ovulatory disorder infertility. Epidemiology. 2009;20(3):374-81.

26- Tielemans E, Burdorf A, te Velde ER, Weber RF, van Kooij RJ, Veulemans $\mathrm{H}$, et al. Occupationally related 